\title{
Bioanalysis
}

\section{White Paper on recent issues in bioanalysis: focus on new technologies and biomarkers (Part 3 - LBA, biomarkers and immunogenicity)}

The 2015 9th Workshop on Recent Issues in Bioanalysis (9th WRIB) took place in Miami, Florida with participation of 600 professionals from pharmaceutical and biopharmaceutical companies, biotechnology companies, contract research organizations and regulatory agencies worldwide. WRIB was once again a 5 day, weeklong event - A Full Immersion Bioanalytical Week - specifically designed to facilitate sharing, reviewing, discussing and agreeing on approaches to address the most current issues of interest in bioanalysis. The topics covered included both small and large molecules, and involved LCMS, hybrid LBA/LCMS and LBA approaches, including the focus on biomarkers and immunogenicity. This 2015 White Paper encompasses recommendations emerging from the extensive discussions held during the workshop, and is aimed to provide the bioanalytical community with key information and practical solutions on topics and issues addressed, in an effort to enable advances in scientific excellence, improved quality and better regulatory compliance. Due to its length, the 2015 edition of this comprehensive White Paper has been divided into three parts. Part 3 discusses the recommendations for large molecule bioanalysis using LBA, biomarkers and immunogenicity. Part 1 (small molecule bioanalysis using LCMS) and Part 2 (hybrid LBA/LCMS and regulatory inputs from major global health authorities) have been published in volume 7, issues 22 and 23 of Bioanalysis, respectively.

The 9th Workshop on Recent Issues in Bioanalysis (9th WRIB) was held in Miami, Florida on April 13-17, 2015 with participation of over 600 professionals from pharmaceutical and biopharmaceutical companies, biotechnology companies, contract research organizations and regulatory agencies worldwide. The workshop included three sequential core workshop days and six specialized training sessions that together spanned an entire week in order to allow exhaustive and thorough coverage of major issues in bioanalysis, biomarkers and immunogenicity. Like the previous workshops, the 9th WRIB was specifically designed to facilitate sharing, reviewing, discussing and agreeing on approaches to address the most current issues of interest in both small and large molecule bioanalysis using LCMS, hybrid LBA/LCMS and LBA approaches. An in-depth focus was on biomarkers, immunogenicity and emerging technologies.

The actively contributing chairs in the 2015 edition of the WRIB were Dr. Eric Fluhler (Pfizer), Dr. Jan Welink (EMA/Dutch MEB), Dr. Brad Ackermann (Eli Lilly), Dr. Fabio Garofolo (Angelini Pharma), Dr. An Song (Genentech), Dr. Theingi Thway (Amgen), Dr. Lakshmi Amaravadi (Biogen Idec) and Dr. Heather Myler (Bristol-Myers Squibb).

The numerous regulatory agency representatives who contributed to the 9th WRIB included Dr. Sam Haidar (US FDA), Dr. Susan Kirshner (US FDA), Dr. Brian Booth (US FDA), Dr. Michael Skelly (US FDA), Dr. Nilufer Tampal (US FDA), Dr. Jan Welink (EMA/Dutch MEB), Dr. Olivier Le Blaye (France ANSM), Ms. Emma Whale (UK MHRA), Mr. Stephen Vinter (UK MHRA), Dr. Bärbel Witte (German BfArM), Dr.

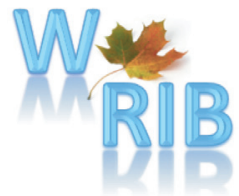

Lakshmi Amaravadi ${ }^{1}$, An Song ${ }^{2}$, Heather Myler ${ }^{3}$, Theingi Thway ${ }^{4}$, Susan Kirshner ${ }^{5}$, Viswanath Devanarayan ${ }^{6}$, Yan $\mathrm{G} \mathrm{Ni}^{3}$, Fabio Garofolo*,7, Herbert Birnboeck ${ }^{8}$, Susan Richards 9 , Shalini Gupta ${ }^{4}$, Linlin Luo ${ }^{3}$, Clare Kingsley ${ }^{10}$, Laura Salazar-Fontana9, Stephanie Fraser $^{11}$, Boris Gorovits ${ }^{12}$, John Allinson ${ }^{10}$, Troy Barger ${ }^{4}$, Shannon Chilewski ${ }^{13}$, Marianne Scheel Fjording ${ }^{14}$, Sam Haidar ${ }^{5}$, Rafiqul Islam ${ }^{15}$, Birgit Jaitner ${ }^{16}$, John Kamerud ${ }^{17}$, Noriko Katori18, Corinna Krinos-Fiorotti ${ }^{12}$, David Lanham ${ }^{19}$, Mark $\mathrm{Ma}^{4}$, Jim McNally ${ }^{12}$, Alyssa Morimoto2, Daniel Mytych ${ }^{4}$, Andre Nogueira da Costa ${ }^{20}$, Apollon Papadimitriou ${ }^{21}$, Renuka Pillutla ${ }^{3}$, Soma Ray ${ }^{1}$, Afshin Safavi ${ }^{22}$, Natasha Savoie $^{23}$, Martin Schaefer ${ }^{21}$, Judy Shih ${ }^{4}$, John Smeraglia20, Michael F Skelly ${ }^{5}$, Jeffrey Spond ${ }^{24}$, Roland F Staack ${ }^{21}$, Bruce Stouffer ${ }^{3}$, Nilufer Tampal $^{5}$, Albert Torri ${ }^{25}$, Jan Welink ${ }^{26}$, Tong-Yuan Yang ${ }^{27}$ \& Jad Zoghbi ${ }^{9}$

*Author for correspondence:

Tel.: +39069 1045277

f.garofolo@angelini.it

Author affiliations can be found at the end of this article.

The views expressed in this article are those of the authors and do not reflect official policy of the US FDA, Europe EMA, Health Canada, France ANSM, The Netherlands MEB, Germany BfArM, Brazil ANVISA, Japan MHLW and UK MHRA. No official endorsement by the FDA, EMA, Health Canada, ANSM, MEB, BfArM, ANVISA, MHLW or MHRA is intended or should be inferred.
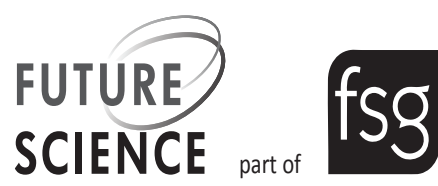


\begin{tabular}{|ll|}
\hline Acronyms & \\
\hline Abbreviation & Definition \\
ADA & Antidrug antibody \\
\hline BMV & Bioanalytical method validation \\
\hline CDR & Complementary determining region \\
DAF & Dual acting F(ab')2 \\
\hline GBC & Global Bioanalysis Consortium \\
\hline HRMS & High resolution mass spectrometry \\
\hline ISR & Incurred sample reanalysis \\
\hline ISS & Incurred sample stability \\
\hline LBA & Ligand binding assay \\
\hline LCMS & Liquid chromatography mass spectrometry \\
MAb & Monoclonal antibody \\
\hline MALDI-TOF & Matrix assisted laser desorption/ionization-time of \\
\hline MOA & flight \\
\hline MRD & Mechanism of action \\
\hline MSR & Minimum required dilution \\
NAb & Minimum significant ratio of titers \\
\hline PD & Neutralizing antibody \\
\hline PK & Pharmacodynamic \\
\hline QC & Pharmacokinetic \\
\hline SDS-PAGE & $\begin{array}{l}\text { Sodium dodecyl sulfate polyacrylamide gel } \\
\text { electrophoresis }\end{array}$ \\
\hline WEC & Size exclusion chromatography \\
\hline
\end{tabular}

Mark Bustard (Health Canada), Mr. Gustavo Mendes Lima Santos (Brazil ANVISA) and Dr. Noriko Katori (Japan MHLW-NIHS).

Each of the three Sequential Core Workshop days was designed to cover a wide-range of bioanalytical topics suggested by members of the community, and included presentations from industry leaders and regulatory representatives, culminating in an open panel discussion between the presenters, regulators and attendees in order to determine the consensus items presented in this white paper.

As with prior WRIB editions [1-9], a significant number of topics were addressed during the workshop and condensed into a series of relevant recommendations. In the present White Paper, the exchanges, consensus and resulting recommendations on 34 recent issues ('hot' topics) in bioanalysis, biomarkers and immunogenicity are presented. These 34 topics are distributed within the following areas:

Small molecules by LCMS:

- Innovations in Small Molecule Bioanalysis (six topics);
- Regulatory Challenges in Small Molecule Bioanalysis (six topics);

\section{Hybrid LBA/LCMS:}

- Innovative Method Development for Biotherapeutics, Biomarkers and antidrug antibody (ADA) (five topics);

- Regulatory Challenges (three topics);

Large molecules by LBA, Biomarkers and Immunogenicity:

- LBA Bioanalytical Challenges (four topics)

- Biomarkers (three topics);

- Immunogenicity (seven topics)

Inputs from Regulatory Agencies:

- Following the recommendations on the above topics, an additional section of this White Paper focuses specifically on several key inputs from regulatory agencies.

Due to its length, the 2015 edition of this comprehensive White Paper has been divided into three parts for editorial reasons. This publication (Part 3) covers the recommendations for Large Molecule Bioanalysis using LBA, Biomarkers and Immunogenicity. Part 1 (Small molecule bioanalysis using LCMS) and Part 2 (Hybrid LBA/LCMS and Regulatory Agencies' Inputs) were published in volume 7 of Bioanalysis, issues 22 and 23 (2015), respectively.

\section{Discussion topics}

\section{LBA bioanalytical challenges}

Unresolved issues in LBA validation from the 2014 White Paper in Bioanalysis

Should hemolysis and lipemia tests be performed for LBA? If not, why are regulatory agencies requesting them? Is incurred sample reanalysis (ISR) really necessary for LBA? Should stabilities be evaluated at actual study sample concentrations rather than in diluted quality control (QC) samples? Do stabilities at QC concentrations really mimic stabilities at incurred sample concentrations? Should additional QC samples be added when sample results are clustered (often due to the nature of the dilutions chosen)? Should dilution QC samples be included in any sample analysis runs? How should differences in data be addressed between two LBA platforms that had been previously cross-validated? 
Issues in developing methods for combination biotherapeutics, bispecific therapeutic proteins \& multifunctional proteins

What are the appropriate drug form(s) to measure for bispecific antibodies? What bioanalytical approach(s) are being used for combination biotherapeutics? Multiple methods are needed to fully understand the properties of these molecules. What are the pros/ cons of Universal LBAs versus Specific LBAs? Which functional domain is involved? What is the best strategy for fit-for-purpose method design and validation? How should the interference on LBA due to binding of biotherapeutic combinations be overcome? How should differential binding affinity for multidomain proteins be overcome? Should all binding sites be taken into consideration?

\section{Impact of biotransformation of biotherapeutics on LBA}

How well do we understand the analyte and applied assays? How do we provide the 'right' information?

Critical reagents \& new stability matters in LBA What is the industry experience with commercially available reagents? What is industry best practice in terms of quality control of LBA reagents? What are regulatory expectations?

\section{Biomarkers}

\section{Commercial kit biomarker characterization \\ \& qualification/validation}

What are the best practices for confirming the specificity of commercially available immunoassays used for clinical biomarker analyses? What level of characterization is needed for exploratory versus confirmatory analyses? Should different immunoassay lots be evaluated in order to support an exploratory versus confirmatory biomarker? Should inter-lot variability be assessed with endogenous and/or recombinant reference standard? If inter-lot variability is observed for in-study analysis, what are the best practices for addressing the impact on biomarker data?

\section{Exploratory versus confirmatory biomarker validation}

What are the challenges with implementing the 2013 FDA Bioanalytical Method Validation (BMV) Draft Guidance [10] requirements for biomarker assays, specifically regarding the use of QC samples in the biological matrix of study samples, justification for using a different matrix for QC samples, demonstrating adequate precision and accuracy, and finally, demonstrating specificity and analyte stability under actual

\section{Key terms}

Biomarker: A measurable indicator of a biological state or process, or a response to a stimulus including drug treatment.

Immunogenicity: The ability of a substance, including biotherapeutic substances, to provoke an immune response.

Cut-points: Thresholds that, when exceeded, establish whether a tested sample is scored as ADA positive.

conditions? What if accuracy cannot be demonstrated since analytes are endogenous, heterogeneous and often structurally different from reference standards? How is inconsistent data from validated methods handled when coming from identical samples, labs or kit vendors?

\section{Characterization of reference materials for soluble protein biomarkers}

What is an appropriate reference material for a protein biomarker assay? What are the issues in using recombinant proteins as reference materials? How to characterize a recombinant protein reference material?

\section{Immunogenicity}

Immunogenicity cut-point evaluations

What is the strategy for screening cut-point evaluations when variances are significantly different between assay runs? What is the strategy for confirmatory cut-point evaluations when means and/or variances of percentage inhibition are significantly different between assay runs? What are alternative strategies for confirmatory cut-points? When and how do you evaluate titration cut-points? What is an objective metric for differentiating titer results across samples (e.g., from a patient over time)? How do you establish/justify the suitability/validity of a negative QC for floating cut-points? When and how is cutpoint suitability for the study population evaluated using (in-study) clinical baseline samples? How do you decide whether new cut-points are needed for a different patient population (disease, demographic, etc.)?

\section{Bioanalytical formats \& impact on comparability} for immunogenicity for biosimilars

Attempt to establish consensus in the industry on which assay format to use for assessing immunogenicity of biosimilars: one assay versus two assays approach. Issues with one assay approach include if the biosimilar is used as a capture reagent, it may not be able to bind ADA that are unique to the innovator 
and vice versa. This may create a risk of generating false negative results. Compare ADA detection rates for innovator and biosimilar compounds for different testing strategies. The ADA assay positive control is used to evaluate specificity, sensitivity, drug tolerance, precision and as a system suitability control in-study. For biosimilar/innovator immunogenicity testing, how many positive controls are needed: 1 or 2 ?

Addressing drug/target interference in the ADA \& impact of ADA/target on pharmacokinetic (PK) assays: a circular situation

How does one get out of the circular situation where the drug interferes with ADA and drug target (biomarker) assays and in turn ADA can interfere with PK and drug target (biomarker) assays? Drug target may further interfere with PK and ADA assays. There are also challenges for receptor occupancy (RO) assays.

Immunogenicity of bispecific therapeutic proteins \& multifunctional proteins

Does a consensus exist to use a risk-based approach to develop the testing strategy for complex biotherapeutics? When should a multiple screening assay development approach be considered and how soon in development? Is there a harmonized recommendation from the different health agencies to conduct a fit-for-purpose method strategy to evaluate the immunogenicity of complex biotherapeutics?

\section{Competitive LBA versus cell-based Neutralizing} antibody (NAb) assays

What are the pros and cons of Category 1 (low risk) and Category 2 (high risk) classifications? Where do moderate-risk molecules fit in? What is the Category 1 molecule testing strategy? What is the Category 2 molecule testing strategy?

Unresolved issues on pre-existing antibodies \& immunogenicity risk

How frequent are pre-existing antibodies $(\mathrm{Ab})$ observed? How are pre-existing $\mathrm{Ab}$ managed for clinical IMG reporting? How are pre-existing antibodies assessed for risk to patient?

\section{Use of biomarker activity for immunogenicity} assessments

When can we use an immune response indicator to monitor loss of activity? How much data are required to correlate clinical impact? When might biomarkers be preferable to conventional ADA/NAb testing? When are they appropriate for safety?

\section{Discussions, consensus \& conclusions}

\section{LBA bioanalytical challenges}

Unresolved issues in LBA validation from the 2014 White Paper in bioanalysis

FDA issued a draft guidance [10] to propose revisions to the 2001 BMV guidance [11] and new draft guidance documents for biosimilars and biological products $[12,13]$. These guidances make recommendations on ligand binding assays (LBAs) for pharmacokinetic, pharmacodynamic, biomarker, surrogate efficacy or immunogenicity measurements. Key parts of these documents were thoroughly discussed in the 2014 White Paper in Bioanalysis Part 3 [9]. Several issues were left unresolved and so were brought back to the table during the panel discussions of the 9th WRIB.

Testing for hemolysis \& lipemia effects during LBA method validation

The consensus among panelists and audience was that these tests should be performed either at the method development stage or in validation. Without data, it may be hard to conclude whether hemolysis and lipids will affect the method or not. It is possible that different therapeutic molecules and their respective methods may show differences or different risks for such interference. It was discussed that examples had been observed where variations in matrices impacted final bioanalytical concentration values, one example being hemolysis effects on some insulin assays [14,15].

ISR for LBA

Following the 2009 EMA BMV guidance document [16] and the 2013 FDA draft guidance [10], an evaluation of ISR has been requested by regulatory agencies worldwide. However, the LBA community has continued to question the need to perform ISR. Discussions around ISR failures in LBA highlighted that these may be due to differences in critical quality attributes of reference standards (e.g., potency or purity), matrix differences, operational reproducibility, in addition to analyte matrix stability. For example, cases where changes in matrix $\mathrm{pH}$ affected binding and assay sensitivity were identified during regulatory review of data. Therefore, it is highly recommended to perform the ISR evaluation since it is useful to uncover potential method weaknesses and to enable investigations that are recommended to understand LBA assay performance.

\section{QC samples used to demonstrate stability in LBA}

Since the 2001 FDA BMV Guidance [11] and continuing through the latest finalized BMV guidance released by the EMA [16], low and high QC samples have been the 
usual means of demonstrating sample stability. However, calibration ranges for LBAs are usually narrow and do not cover the concentration range of study samples, hence requiring dilution during sample analysis. Therefore, the question arises whether QC samples used to demonstrate stability should be stored at pharmacokinetic or calibration range concentrations in order to be considered representative of study samples. In the 2014 White Paper in Bioanalysis [9], it was stated that in some cases, equilibrium shifts between bound and unbound forms of the drug following sample dilution may impact the form of the analyte being measured. Therefore, for certain forms of analytes with suspected degradation sites, it was recommended that stability evaluations be assessed at the concentrations of study samples (at pharmacokinetic concentrations) since the stability samples should mimic the range of study samples.

\section{The addition of extra QC samples during sample analysis}

Two scenarios were the focus during review of this topic. The first is when study sample concentrations are clustered in one area of the calibration range. As previously discussed [9], this is often related to selection of dilution factors for sample analysis, and the clustering of results near the middle of the calibration range can actually be seen as the ideal situation. Since the calibration range is typically narrow, it is unlikely that calibrator or QC sample failure in one section of the curve would still result in a passing batch; so additional QC concentrations may be unnecessary. This position was confirmed again during this year's panel discussion, with a comment that extremely narrow calibration ranges can force results toward middle concentrations. The second scenario involved adding dilution QC samples to sample analysis batches. It has been recommended that this would only be required when needed by a specific assay or as required by lab operational procedures.

\section{LBA platform cross-validation}

The final remaining 2014 WRIB topic regarded differences in concentration results between two LBA platforms that had been previously cross-validated. It was clear that an investigation would be needed to explore the reasons for the differences. Finally, the scientific reasons for choosing one assay platform over another should be documented.

Issues in developing methods for combination biotherapeutics, bispecific therapeutic proteins \& multifunctional proteins

Assays that are well characterized and reliable are needed to evaluate pharmacokinetics, toxicokinetics and bioequivalence. Protein therapeutics have become increasingly important in medicine in almost

\section{Key term}

Bispecific therapeutic proteins: Proteins that are composed of fragments of different specificity thereby binding to two different types of antigen.

every therapeutic area and new therapeutic approaches include biotherapeutic combinations, novel structures such as bispecific antibodies/therapeutic proteins and multifunctional proteins. These new classes or combinations of compounds could present unique challenges for drug development including bioanalysis. With over 60 formats of bispecific therapeutic proteins $[17,18]$, only a very small set of examples are discussed below.

Many companies are researching the same indications with the goal of developing best-in-class biotherapeutics in order to increase the benefits to patients taking their medications. There are many strategies to enhancing the performance of the biotherapeutics. For example, one can modify existing properties, by engineering the $\mathrm{Fv}$ or $\mathrm{Fc}$ domains in order to affect binding affinities, or by endowing the antibodies with new capabilities in the form of bispecific antibodies or antibody-drug conjugates. One could also use them in combination with other antibodies or nonantibody protein therapeutics, or small molecules. Each strategy (adding new capabilities to a single molecule vs combination therapy) has its advantages and disadvantages.

Bispecific antibodies/therapeutic proteins introduce new bioanalytical challenges with regard to analyzing the active drug form during PK evaluations. Such methods double the reagent requirements for ligand binding assays as compared with regular antibodies because both targets or anti-idiotypes are needed to detect the active drug form. However, biotransformation may pose a challenge for the quantification of bispecific $\mathrm{F}(\mathrm{ab})_{2}$, antibodies or other forms. For dual acting $\mathrm{F}(\mathrm{ab})_{2}$ antibodies, challenges are similar to those seen when using regular monoclonal antibodies.

It is recommended that due to these bioanalytical challenges, there is a real need to understand the analyte being assayed and the different possible forms in vivo and to develop an appropriate bioanalytical strategy. Further discussions and updated recommendations are planned at the 2016 WRIB on PK assay strategy recommendations for bispecific therapeutic proteins and multifunctional proteins since industry and regulators experience in this arena is still new and limited.

\section{Impact of biotransformation of biotherapeutics on LBA}

It has been discussed and agreed that deamidation of asparagine (Asn) residues is one of the most common chemical degradation pathways of recombinant thera- 
peutic proteins, including monoclonal antibodies. This may occur during production and storage, under formulation conditions, but also happens under physiological conditions, in vivo, after administration to animals or humans. Deamidation of Asn is considered a critical quality attribute when it impacts certain characteristics, especially the biological activity or binding properties of a therapeutic.

A case for which a publication is being prepared was discussed involving the identification and characterization, both biophysical and functional, of Asn deamidation in the complementarity determining region of a monoclonal therapeutic antibody [Емrich T ET AL. IN-VITRO AND IN-VIVO CHARACTERIZATION OF CDR DEAMIDATION OF A MONOCLONAL therapeutic antibody (2015), Manuscript in preparation]. Deamidation at this specific site renders the molecule functionally inactive, unable to bind its respective target. Interestingly, extensive biochemical and functional characterization using a broad panel of analytical and bioanalytical methods showed a completely different kinetic behavior of this modification during storage of the drug product, in formulation buffer, in vitro, compared with degradation under physiological conditions, in vivo.

It is important to understand the nature of the analyte that is quantified in the applied bioanalytical assay. The bioanalytical strategy should enable appropriate decision making and interpretation during PK/pharmacodynamic (PD), safety and other clinical studies. It is recommended to develop an appropriate bioanalytical strategy to allow a differentiation between drug elimination and conversion for a meaningful interpretation of PK behavior and accurate determination of active drug exposure in patients. Moreover, consensus was reached that in order to come up with the most appropriate bioanalytical strategy, multiple technologies and tools, and various approaches including qualitative and quantitative methods may be needed to understand the impact of biotransformation of biotherapeutics on function.

Ideally the quantified analyte reflects active drug, as only active drug quantification enables meaningful determination of exposure in case of biotransformation of the drug into an inactive metabolite. Measuring inactive drug may be regarded as supplementary information only and, as such, is not recommended as the quantitative assessment of drug levels. Highly selective immunoassays, using specific well-characterized anti-idiotypic antibody preparations that are able to discriminate between deaminated and nondeaminated variants of the drug, are valuable tools for assay setup and highly selective quantification of active (nondeamidated), inactive (deamidated) and total drug in blood samples.
Critical reagents \& new stability matters in LBA Ligand-binding assays are frequently applied and considered the gold standard in the bioanalysis of large molecules, for example, for the quantification of therapeutic proteins and for immunogenicity testing. Besides the selection of the appropriate testing platform and assay format, adequate quality of critical reagents used in the ligand-binding assay is of key importance for establishing a robust and reliable performance of the bioanalytical assay. Critical reagents are those used for capture and detection of the biotherapeutic; some commonly used critical reagents include biotinylated (Bi) antibodies (capture Abs or detection Abs), sulfotag labeled and digoxygenylated antibodies (detection Abs) [19].

In addition to the assay design, selection, production and characterization of critical reagents, reliable longterm supply is mandatory for the support of bioanalytical work during the life-cycle of a project [20-22].

It has been agreed that characterization of reagent functionality should include an early assessment of binding properties (cross-reactivity to matrix components), binding kinetics and any altered binding issues due to, for example, labeling or storage. Biophysical characterization of reagents should include identity, concentration, storage conditions and stability information. Purity information may be relevant for trouble-shooting. Documentation of these properties is typically in the form of a certificate of analysis or a technical data sheet $[19,23]$.

The use of commercially available reagents is one way to obtain reagents, however industry experience with these is mixed. If commercial reagents are used, it is recommended to try to obtain well-characterized reagents or characterize them in-house to ensure lot-tolot consistency and long-term supply. The characterization and qualification of critical reagents should follow a fit-for-purpose approach predefined in an SOP based on the needs of the results of the assay (e.g., exploratory vs confirmatory) [19,20].

\section{Biomarkers}

Biomarkers are commonly utilized to demonstrate therapeutic safety, target engagement, pathway modulation and as predict the course of a disease and/or response to therapeutic intervention. As such, biomarker data are used to inform key milestones in the drug development process. Blood collection is a convenient and easy way to monitor biomarkers and their presence and levels are often examined in a variety of biological fluids using immunoassay based techniques. Many pharmaceutical companies develop immunoassays de novo in-house, however the plethora of commercially available immunoassays offers an attractive and cost affective option 
for identifying circulating biomarkers. This can enable the investigation of more biomarkers than internal resources can support. Using in vitro diagnostic kits for research purposes requires additional adjustments to the method and fit-for-purpose testing to demonstrate that the results being generated are reliable and answer the question being asked $[24,25]$.

\section{Commercial kit biomarker characterization \& qualification/validation}

Good immunoassay tools are needed for testing biomarker hypotheses. There are predictive biomarkers, which may identify patients who are more likely to develop a disease or respond to the drug (patient stratification). Pharmacodynamic biomarkers demonstrate target and pathway engagement by the drug and can be used to assess the relationship between drug pharmacokinetics and biological response. Finally, there are prognostic disease biomarkers, which can inform researchers and clinicians on the future progression of a particular disease. Understanding the intended end use of the biomarker is a key driver in choosing the right assay for determining the concentration of the biomarker being assessed. Researchers justifiably do not want to reinvent the wheel, and so often look to commercially available research-use-only or diagnostic kits for their drug development programs [26].

The level of assay characterization needed between exploratory versus confirmatory analyses is driven by a risk-based assessment taken within the drug development programs of company. The decision is based on the intended use of the data and should be driven by the clinical utility of the results. The level of bioanalytical validation may increase or decrease accordingly [27].

\section{Specificity}

It is essential to understand the value and limitations of the kits and kit reagents during kit characterization studies. The presence of a signal and sensitivity specifications as determined by the vendor are not enough to confirm that the kit is detecting the biomarker of interest. The source and bioanalytical characteristics of the assay reagents provided are typically unknown; kit users should contact the vendor and request reagent specificity and epitope information if it is not provided. If possible, it is recommended to conduct competition studies (i.e., using excess antibodies or biomarker binding proteins to reduce assay signal to $\leq$ LLOQ) and test reference standards from multiple kit vendors or commercial sources to verify the specificity of the assay.

\section{Inter-lot variability}

If only one kit lot will be used for the entire study, an assessment of inter-lot variability is not required. How- ever, if multiple lots are needed in a study, it is recommended to test as many lots as reasonably possible during development and assay validation to assess the potential impact of inter-lot variability on study data. It is worth noting that although kit lot numbers may change due to modifications in noncritical reagents such as kit buffers, critical reagent lots may not have changed. In such cases, an inter-lot variability study may not be necessary. It should be confirmed with the vendor, if possible, whether different lots of critical reagents are available and these should be used in the end-users' assessment of inter-lot variability. If multiple lots of assay kits are likely to be required to support the clinical program, a bridging strategy should be developed to mitigate the potential impact of lot-to-lot variability. These issues are study dependent and should be considered during the risk assessment.

If inter-lot variability is observed during the lot bridging assessment, an initial practical option is to contact the vendor(s) for replacement of the reference standard or critical antibodies used. If this is not possible, a statistical assessment of the impact of the variability on the clinical data may provide the best fallback approach. Correction factors may be used with caution and when used, it is important to assess linearity or response using sufficient QC samples across the assay range. It is also important to understand the nature of the most appropriate QC samples when assessing linearity - endogenous or recombinant sources of the biomarker. Parallelism testing should be performed to confirm that recombinant material is comparable to endogenous analyte.

\section{Reference standards}

If possible, it is best practice to use the same reference materials during validation and sample analysis. Additionally, if internationally recognized reference standards are available, these should be prioritized.

\section{Exploratory versus confirmatory biomarker validation}

Biomarker assays broadly belong to one of two categories based on the intended use of the data. The first category - exploratory biomarker assays - is used to generate data for internal decision-making and does not drive label claims. This data can help in understanding the pharmacodynamics, mechanism of action of the drug or can be more broadly used for hypothesis generation. Assays for these types of biomarkers can vary in quality and robustness from in-house developed assays to research-use-only commercial kits to FDA approved diagnostic kits, validated for specific uses. Methods for exploratory biomarkers can be used during the discovery Phase I profiling studies (requiring 
modest analytical evaluation) or during Phase II-III studies determining proof-of-concept or dose optimization (requiring more rigorous analytical evaluation). Confirmatory biomarker assays, the second category, generate data supporting pivotal determinations of efficacy or label dosing instructions, making it critical to ensure the integrity of the data. These biomarkers are typically used to support applications for Phase IIIII studies determining proof-of-concept, dose escalation studies (requiring modest analytical evaluation) or label claims, patient stratification and other studies included in drug applications (requiring rigorous analytical evaluation) [10].

These categories are not always distinct during the drug development process and the levels of assay validation required may vary (full vs partial validation) depending on the type of assay employed. In addition it is also important to understand the class of analytical assay depending on the measurand and type of reference material available. The first type can be termed as a definitive quantitative assay, which uses well-characterized reference standards representing the biomarkers being tested to create a response-concentration profile to allow for quantification of unknown samples. The second type is the relative quantitative assay where the reference standard is not as well-characterized, pure or closely representative of the biomarker. The third type is often termed semi-quantitative and characterized by the lack of a calibration reference standard, but the production of a continuous readout, for example, cell numbers in flow cytometry. The final class can be a qualitative assay, where biomarker concentrations are not measured with any accuracy, but results are nominal or ordinal in nature [27]. No matter the type of biomarker being studied or the hypothesis being tested, there are general challenges that need to be addressed by all biomarker assays. These include, for example, purified or recombinant reference standards that are not necessarily representative of the endogenous analyte, limited availability of reliable critical reagents, extended timelines needed to generate new reagents, as well as challenges of using in vitro diagnostic kits for matrices and disease state samples for which they were not developed or approved for use. For the majority of protein biomarkers, the kit reference standards do not represent the endogenous biomarker. Therefore, these assays are deemed relative quantitative or qualitative.

The draft FDA guidance for BMV issued in 2013 [10] touched on the subject of biomarkers. Consensus was reached that, due to a variety of challenges outlined above, developing and validating a biomarker assay cannot always meet the standards of a PK/drug assay. The 2013 FDA draft guidance requires the use of QC samples in the same biological matrix as study samples and every effort should be made to use matrix QC samples as part of assay acceptance for confirmatory assays. Best practice is to have at least one matrix QC (low, medium or high) in the same matrix as the study samples. If endogenous analyte concentrations are not high enough, spiked QCs may be used. If the matrix is rare or it is difficult to obtain disease state matrix, then the use of a different or surrogate matrix for QC samples should be justified.

It was agreed that stability testing should be performed in both assay buffer, to determine the stability of QC samples, and sample matrix under the actual conditions that will be used during sample analysis. Matrix stability should be tested with matrix QC samples, however as discussed above, these may not always be available during validation. In these cases, sample stability can only be evaluated during a study on study samples obtained with informed consent. A demonstration of adequate precision and accuracy is a requirement of the guideline. However, if true accuracy cannot be demonstrated because analytes are endogenous, heterogeneous and often structurally different from the reference standards, the impact of this during the drug development process is considered to be minimal because biomarker methods typically fall under relative quantitative or qualitative methods classes, where the assay simply determines the relative amounts of the biomarker against a baseline sample (if available) or another group of samples tested at the same time. When considering confirmatory biomarker assays, the matrix QC samples should cover the analytical range, if possible.

As consensus, it was agreed that despite all best efforts to use reliable and robust methods for sample analysis of biomarkers, there are occasions when study sample testing may need to be repeated, increasing the possibility of having inconsistent data from identical samples assayed in the same lab using identical kits. If the sample is analyzed using a single analyte assay, there should only be one final, reliable result that meets predefined acceptance criteria. If multiple analytes are tested in the context of a multiplex assay and samples need to be reanalyzed for only certain analytes, it is recommended not to regress any analyte data that have previously passed. Acceptance of the first set of data should be predefined in SOPs.

\section{Characterization of reference materials for soluble} protein biomarkers

Bioanalytical assays are based on some fundamental assumptions; that we know what we want to measure, we know what is being measured, and that the reference material represents the target analyte. However, in biomarkers assays, these assumptions are not always true. It is not always clear a priori which form of a biomarker is the most important to measure (e.g., isoforms, 
truncations, complexes). What is being measured in some methods is not always what we intended to measure. Careful selection and characterization of reference materials is critical to understand what the assay is really measuring, and to ensure acceptable assay performance.

Soluble proteins in human circulation are suitable targets for biotherapeutics since they are easily accessible. Free and total soluble protein biomarker assays can provide good measures for target engagement and pharmacodynamics. As for most biomarker assays, reference standards that properly represent endogenous soluble proteins are difficult to find. The parallelism test should be used to demonstrate that the dilution response curve for samples with endogenous analyte is parallel to the dilution response curve of the reference material. When parallelism cannot be established, a fit-for-purpose strategy should be applied to use reagents that, although different from endogenous analytes, are able to provide relatively quantitative measures of change in analyte levels.

Most often, recombinant proteins are used which resemble the endogenous analytes in many aspects such as post-translational modifications, physico-chemical state and biological interactions. However, these recombinant protein reference materials may not be able to fully represent endogenous analytes, since the endogenous analytes are often heterogeneous and undergo a variety of post-translational modifications such as glycosylation, phosphorylation and oligomerization not present in the prokaryotic expression system. Therefore, biomarker assays using recombinant proteins as reference materials are relatively quantitative in nature [27]. It should also be noted that the issues described in this paragraph are not unique to biomarker assays. Immunogenicity assessments also lack reference material and ADC analytes are not necessarily representative of the reference material either.

It is recommended that careful selection and characterization of reference materials is critical for understanding the specificity of the assay (what the assay is really measuring), and for ensuring acceptable assay performance and appropriate data interpretation.

Reference standards should be selected based on the biological properties of the analyte of interest, their stability, their availability in suitable quantities and their well-characterized properties. The characterization process may include but is not limited to:

- Identity tests to determine molecular weights and peptide mapping using High resolution Mass Spectrometry or Matrix Assisted Laser Desorption/ Ionization-Time of Flight can be performed.

- Purity, carbohydrate content and oligomeric states can be determined using, for example, sodium dodecyl sulfate polyacrylamide gel electrophoresis and size exclusion chromatography techniques.

- Physico-chemical states affecting binding with capture and detection antibodies.

- Potency measurement.

- Content assignment, in other words, mass and specific activity.

- Operational aspects such as short and long term stability under defined storage conditions, lot to lot bridging.

It is acknowledged that the characterization parameters cannot be accomplished using commercially available reference standards, as often the quantity of material is limited.

\section{Immunogenicity}

ADA immune responses to protein therapeutics are of concern because ADAs can impact the PK, PD, safety and efficacy of protein therapeutics by inhibiting the function of endogenous proteins leading to deficiency syndromes; altering the pharmacokinetics and pharmacodynamics of drugs, causing hypersensitivity type adverse events; and/or neutralizing the efficacy of the drug. Therefore, it is crucial to establish the potential relationships between ADA status versus PK, PD, safety or efficacy during clinical development. ADA screening, confirmation, titration and neutralization activity assays are implemented to monitor and characterize the ADA responses.

\section{Immunogenicity cut-point evaluations}

Establishing appropriate cut-points based on sound statistical principles combined with an understanding of the biological and bioanalytical issues help ensure that assays reliably detect ADA with adequate sensitivity. The cut-point evaluation strategy has evolved considerably over the past decade via the seminal white-papers on the design and validation of immunogenicity methods [28,29], and refined further over the years by similar consensus groups of experts as in the USP Chapters $<1106>$ on immunogenicity screening and neutralizing antibody assays [30,31]. At this workshop, a simplified version of the flow-scheme proposed originally in the validation white-paper [29] was discussed.

The use of floating cut-points is recommended for screening cut-point evaluations when means are significantly different between the assay plates/runs, whereas when variances are significantly different the use of a dynamic cut-point is recommended in the validation white-paper [29]. However, this is usually impractical as it requires a separate evaluation of a cut-point in each 
assay run using several individual drug naive subject sera, and hence lowering the sample testing throughput. A work around this scenario is explained in the USP chapter [31] where the sources of assay variance may be investigated for further optimization when feasible. If variance heterogeneity cannot be resolved, the USP chapter [31] recommends the floating cut-point method to be used by pooling the variances across the assay runs. It has also been discussed that if only one or two runs are responsible for the overall variance heterogeneity, then such runs may be eliminated before pooling the variances of the data from the other runs for the floating cut-point evaluation. Based on the experience of the panel, it was also noted that the use of analyst or instrument specific cut-points although recommended in the validation white-paper [29] should be avoided.

An inherent assumption made in the use of a floating cut-point method for the screening cut-point evaluation is that the negative control used for setting the correction factor trends (changes) in the same direction as the individual drug naive subject sera across the assay plates and runs. This assumption may be verified by plotting the average of negative control results versus the average of individual subject sera results across the validation runs (18 plates, three plates/run from six runs). Appropriate statistical tests may be conducted for objective assessment such as testing whether the slope is significantly different from one and/or using a criterion on the correlation. If the negative control does not trend in the same direction as the individual subject sera, the use of alternative negative controls was discussed as well. This may include buffer or serum diluent or the use of subject sera pool from a population that more closely mimics the study population. This topic requires further research via case-study examples, and may be discussed further at the next WRIB.

It was agreed that the strategy of many in the industry for the confirmatory cut-point evaluation, even when the means and/or variances of percentage inhibition are significantly different between assay runs, is to use a fixed cut-point that is statistically determined by spiking drug in naive, ADA negative subject samples [29]. The use of negative QC samples with spiked drug for possible use of a floating cut-point method was proposed. As this idea has not been tested, the recommendation is still to use the fixed cut-point method for the confirmatory assay.

Alternative strategies for confirmatory cut-points such as the use of mock low positive samples may be possible and have been discussed extensively in a prior publication [32].

The use of titration cut-points was then discussed. This cut-point is necessary only when the screening cut-point is too low, for example, if it falls on the lower plateau of the positive control dilution curve. The same data generated for the screening cut-point evaluation are typically used for the titration cut-point evaluation, where instead of targeting a $5 \%$ false positive rate, a much lower error rate such as $0.1 \%$ is targeted.

The recommended objective metric for differentiating titer results across samples (e.g., from a patient over time) is the minimum significant ratio of titers, derived from validation runs with the positive control in sensitivity experiments [30]. This is especially relevant if the titer results for confirmed ADA positive samples are determined via interpolation. However, if end point titers are reported instead of interpolation, a fourfold difference may be considered as significantly different if twofold serial dilutions are used and a ninefold difference as significant for threefold serial dilutions [30-31,33].

The suitability of using the prestudy validation cut-point factor for testing clinical study samples was discussed. Based on the simulation studies done for typical screening cut-point evaluations for a balanced design described in the validation white-paper [29], the false positive rate for a screening cut-point that is targeted around a 5\% false positive rate is expected to vary between 2 and $11 \%$. Therefore, if the false positive rate of the in-study baseline samples is too low $(<2 \%)$ or too high (e.g., $>11 \%)$, it is recommended that the means and variances of the log-transformed ratio of individual subject sera to negative control from the validation (prestudy) and clinical study baseline (in-study) be compared first. If only the means of these ratios are significantly different, use the variance from the validation along with the mean of the ratios from the in-study baseline samples to adjust the cut-point factor accordingly. If the variances are different, the in-study baseline may be used to calculate a new study-specific cut-point correction factor. This is reasonable to do as long as baseline data are available from at least 50 subjects, tested over at least two runs and by at least two analysts. Each sample may be tested only once, but as long as the data are cumulatively generated across multiples runs and analysts, the overall variability evaluated for the study-specific cut-point calculation will appropriately reflect both the analytical and biological variability. One limitation is that it is not possible to break down the analytical and biological variability separately, but this is not necessary at this stage because this has already been assessed and characterized in detail during the prestudy validation.

This study-specific cut-point evaluation is generally feasible with most Phase II and later-phase clinical studies, but not usually in preclinical studies and 
most Phase I studies. For smaller studies such as Phase I, re-evaluation of the cut-point using a larger number of subjects may be necessary when feasible.

When deciding whether new cut-points are needed for different patient populations (disease, demographic, etc.), the log-transformed ratio of individual subject sera to negative control from the prestudy validation should be compared with the ratio results from the new population. Similar to the recommendation described above for determining the need for a studyspecific cut-point factor, the means and variances of the ratio results from these populations should be compared. If the variances are significantly different, a new cut-point factor should be evaluated for the new population. If only the means are different, the same cut-point factor may be used with a new negative control pool created using subjects from the new population.

\section{Bioanalytical formats \& impact on similarity for immunogenicity for biosimilars}

Biosimilars have become of high interest recently and a number of regulatory guidance documents have been published stating that the demonstration of clinical immunogenicity similarity is a key element in establishing biosimilarity between the biosimilar and reference drugs. However, demonstration of immunogenic similarity between a biosimilar and the reference drug is challenging due to the fact that ADA assays are qualitative by nature, unique to each drug, and proprietary to each drug sponsor. Also, there is no consensus in the industry on whether the innovator's ADA assay, the biosimilar assay, or both should be used to assess immunogenic comparability.

There has been much discussion about whether the best option is to use a two assay approach or the one assay approach. One assay might risk the appearance of higher immunogenicity with the biosimilar. It should be mentioned that if using one assay, it is imperative to use the biosimilar and not the reference product to generate binding reagents. There are as many challenges with both approaches as there are arguments that both approaches are valid. It should be noted that FDA recommends that the proposed product and the reference product should be assessed in the same assay with the same patient sera whenever possible [34]. With accumulating experience with time, a unified data-driven recommendation is expected to emerge.

Analytical approaches employed in the characterization of biosimilar products are expected to detect immunodominant epitopes. Furthermore, the use of a LCMS approach may be a suitable option for nonmonoclonal antibody drugs if the necessary sensitiv- ity of the assay can be achieved [35]. Ultimately, the impact on clinical safety and efficacy is what matters. It was agreed that this topic should be discussed again at a future WRIB to reach a wider consensus.

Addressing drug/target interference in the ADA \& impact of ADA/target on PK assays: a circular situation

The presence of a drug and/or drug target can cause interference in patients' ADA and NAb assessment, resulting in potential false negative or false positive results. The degree of interference is related to the assay platform, patient specific factors (disease) or presence and levels of drug or drug target in the sample. In the ADA assays, false negatives with drug interference and false positives with target interference are possible. In the NAb assays, both false positives and false negatives are possible due to drug and target. Different approaches have been used to mitigate both drug and target interferences; these include acidification, blocking/binding agents and sample pretreatment to remove drug or target [36]. However, sample manipulation has the potential to introduce new artifacts. Regardless of the approach, it is imperative to have a good understanding of the ADA assay and to interpret the data carefully.

It was agreed that a circular situation is created where the drug and target can interfere with the ADA, the target and ADA can interfere with the PK assay and the drug can interfere with the target assay. Data can become difficult to interpret if you have multiple interferences without a thorough understanding of assay caveats. Conversely, understanding the physiological binding events through integrated bioanalytical assay design is important so that this understanding can be incorporated into pharmacometric modeling tools and thus can be highly informative as to the pharmacological state of the patient.

In the case where ADA interferes with PK and drug target assays [37,38], the first step is to understand if free drug (active drug) pharmacokinetics are most relevant because the approach used may depend on the class of therapeutic being tested. It is important to be able to explain the drug clearance. Additionally, if a PD marker is available, it may provide the most relevant information related to efficacy results.

Elimination of drug interference and inter-patient variability through standard LBA mechanisms is encouraged. If this is not possible, it is imperative to have a good understanding of the ADA assay including the immunogenicity risk assessment and mitigation plan so as to interpret the data accurately across functions (bioanalytical scientists to clinical pharmacologists and clinicians). 
Immunogenicity of bispecific therapeutic proteins \& multifunctional proteins

Bispecific therapeutic proteins and multifunctional proteins represent a new class of biotherapeutics used to treat different conditions. These molecules consist of more than one domain that may or may not be involved in the mechanism of action of the drug; examples of these biotherapeutics include Fc fusion proteins, bispecific antibodies, antibody-drug conjugates and pegylated proteins. Similar to any other therapeutic protein, these complex molecules have the ability to elicit an immune response once administered to patients. Evaluation of ADAs against these biotherapeutics represents unique challenges.

It was agreed that bispecific therapeutic proteins and multifunctional proteins (including ADC) may require different considerations. Bispecific immune modulating modalities, along with other biotherapeutics not described herein, may require additional mechanistic characterizations associated with immune responses in addition to ADA testing. These may include monitoring cytokine release and other immune system modulation events [39].

As with all biotherapeutics, bispecific therapeutic proteins that have complex molecular structure and multiple mechanisms of action require an adequate risk assessment evaluation and testing strategy. Classification of risk level (low, medium, high) should be justified in a risk assessment and mitigation plan. The testing strategy will follow the rationale prospectively defined in this plan and will evolve as clinical data are procured.

Screening and confirmatory assays should minimally be performed against the intact bispecific therapeutic proteins per conventional testing strategies. Testing for specificity against the functional domain may be justified as the incidence and clinical impact merits. Specificity testing in the confirmatory tier versus having fully independent domain-based assays is case dependent. Reagents for each domain will be required for adequate domain specificity testing.

Consensus was reached that there are no harmonized recommendations from the different health agencies yet to conduct a fit-for-purpose method strategy to evaluate the immunogenicity of complex biotherapeutics. Hence, it is recommended that each strategy should be evaluated on a case-by-case basis, as previously discussed [40].

\section{Competitive LBA versus Cell-based NAb assays}

Cell based and non-cell based assays can be used for the detection of NAbs in the immunogenicity testing workflow. Industry standards regarding this testing have been evolving for 10 years, resulting in a need for a consistent NAb assay selection strategy across the industry and all regulatory agencies. The most recent proposal of a risk based testing strategy [41] was thoroughly discussed but no consensus was reached due to very different points of view.

The Kloks paper categorizes therapeutic molecules bearing low or high risk due to immunogenicity as Category 1 and 2, respectively. The strategy for risk-based testing for Category 1 (low risk) molecules is proposed as follows in the paper:

- Phase I Single Dose: Event-driven ADA testing of collected samples, no NAb testing or characterization is needed;

- Phase I Multi-Dose: At least baseline and end-ofstudy sampling and testing, no NAb testing or characterization is needed;

- Phase II/III: Frequent sample collection;

- Testing of at least baseline and end-of study samples, $\mathrm{NAb}$ testing and any characterization is optional;

- Sample testing to be performed in a batch wise manner at the end of study.

The strategy for risk based testing for Category 2 (high risk) molecules is proposed as follows in the paper:

- Phase I Single Dose, multi-dose, Phase II/III: ADA and NAb testing (PD evaluation is also an option);

- Characterization may be performed if added value (at baseline and end of study);

- Sample testing to be be performed in a timely manner.

The discussions led to a disagreement with the manuscript recommendations for the Category 1 molecules. In fact, it was recommended that ADA samples need to be collected and periodic testing is highly recommended even for low risk molecules to fully characterize the immune response to the molecule in the clinical population of interest at all stages of development. However, there was agreement with the manuscript recommendations for high risk (Category 2) molecules. It was noted that immunogenicity testing for high risk molecules should follow the conventional scheme that includes testing for binding ADAs, NAb and any other characterization (if needed) that should occur as soon as possible.

\section{Unresolved issues on pre-existing antibodies \& immunogenicity risk}

ADAs are understood to be a potential risk to patient safety with possible impact to efficacy. As such, ADAs 
are routinely monitored during clinical trials [42]. Preexisting ADAs that cross-react with a biotherapeutic drug candidate are often observed during immunogenicity assessment. They can include heterophilic species from the natural antibody population (e.g., rheumatoid factor) [43], antibodies produced as an adaptive immune response to environmental antigens, and can include antibodies against endogenous proteins reported to play a role in immuno-modulatory activities (e.g., anticytokine Abs) [44]. The reactivity of pre-existing antibodies and any related boosting of titer due to therapeutic administration is critical to understanding how pre-existing antibodies can impact patient safety and efficacy $[9,29]$.

Data are still being collected across the industry to gain a better understanding of the prevalence of pre-existing antibodies. Multiple scenarios have been observed by attendees, such as a low prevalence with low titer through to a high prevalence with high titer. Observations on the impact of pre-existing antibodies on PK, safety and efficacy are also variable.

Clinical immunogenicity reporting is managed for pre-existing antibodies by employing patient stratification to independently report boosting and seroconversion events [29]. 'Boosting' events are generally defined as responses twice a two- or three-fold dilution scheme, equating to a minimum of four- to nine-fold increase in titers (typical scenario of $+/$ - one-fold dilution considered acceptable assay variability). Titers are generally reported as the reciprocal of the dilution. For example, a sample with a baseline pre-existing titer of 32 is serially diluted using a two-fold dilution scheme. A boost in titer of 128 would be considered a significant increase, whereas a titer of 64 (one two-fold dilution tube) would be viewed within assay variability and not considered a meaningful change. More sophisticated statistics can be implemented as needed and is most useful if there is supporting clinical data that correlate with clinical impact. Drug interference can impact titer and should be considered when defining boosting criteria.

It was agreed that patient exclusion based upon preexisting antibody presence is an unusual practice and is not recommended. However, there are examples of patient exclusion criteria pertaining to patient disposition that are not completely independent from immune status. In a specific case study discussed [45], it was noted that cryoglobulinemia, lymphoma and pre-existing autoimmune disease were exclusionary criteria in the noted interferon-based clinical studies. Pre-existing antibody status, PEG and interferon $\lambda$ - or interferon $\alpha$-specificity, was not included in the enrollment exclusionary criteria. Direct characterization of ADA during the course of therapy was conducted. Whether to include pre-existing antibody status in the exclusion criteria should be considered based on the knowledge of drug product and safety assessment and should be incorporated into the immunogenicity risk assessment approach.

\section{Use of biomarker activity for immunogenicity assessments}

Standard immunogenicity assessment includes the detection of binding and neutralizing ADAs. The presence of ADA may impact potential loss of response to the drug. Thus, ADA may influence disease activity through limiting the PK and/or PD of the drug $[46,47]$. It was agreed that monitoring of $\mathrm{PK}, \mathrm{PD}$ and $\mathrm{ADA}$ (and its impact) may provide a more comprehensive and personalized approach for disease management. In addition, characterization of a robust ADA response (titer, neutralization/bioactivity) can also offer insight into opportunities for early detection and more targeted monitoring of a clinically meaningful ADA response in patients at risk.

Use of immune response indicators can be considered to monitor loss of activity when ADA is of moderate to high incidence and well characterized to know that ADA indeed have an impact on efficacy and/or safety [48]. This generally occurs during late stage or post-market considerations to inform treatment decisions (e.g., increase of IgG4 in relation to antibody-mediated PRCA) [49].

If there is a robust PD biomarker related to efficacy/MOA it may be more appropriate to correlate PD data to clinical impact. However, this poses a significant logistic hurdle where the biomarker must be clinically validated and may require greater resource commitment than a NAb assay. It was recommended to have a conversation with the regulatory agency to develop a feasible strategy and successful examples of this do exist (e.g., IFN- $\beta$ and MxA).

\section{Recommendations}

Below is a summary of the recommendations made during the 9th WRIB.

\section{LBA bioanalytical challenges}

- Unresolved issues in LBA validation from the 2014 White Paper on Bioanalysis

- Hemolysis and lipemia tests should be performed either at the method development stage or in validation since, without data, it may be hard to conclude whether hemolysis and lipids will affect the method or not.

= ISR for LBA assays is useful to help uncover potential method weaknesses and investigations 
are appropriate to understand LBA assay performance. Hence, it is recommended that ISR should be conducted.

- In addition to regular QC samples, stability evaluations should be considered at the pharmacokinetic concentrations mimicking the range of study samples as much as possible as stability at Cmax levels may not always be represented by low level QC samples. In the case of 'freedrug' assays, further experiments may need to be considered to demonstrate stability of binding equilibria and appropriate sample dilutions.

= The addition of extra QC sample concentrations during analysis when study samples are clustered in one area of the calibration range may be unnecessary when the calibration range is narrow.

= The addition of dilution QC samples to sample analysis batches is not needed as routine procedure. However, dilution QC samples can be included if it is required by lab operational procedures or for pipetting verification.

= When there are differences in concentration results between two LBA platforms that had been previously cross-validated, an investigation would be needed to understand the reasons for the differences. The reasons for choosing one assay platform over another should be documented.

- For bispecific therapeutic proteins and multifunctional proteins, there is a real need to understand the analyte being assayed and the different possible forms in vivo to come up with an appropriate bioanalytical strategy. Further discussions are planned at the 2016 WRIB since industry and regulator experience in this arena is still evolving.

- Ideally the quantified analyte reflects active drug. Measuring inactive drug may be regarded as supplementary information only and, as such, is not recommended as the only quantitative assessment of drug levels. An appropriate bioanalytical strategy should be developed to allow a differentiation between drug elimination and conversion. In order to come up with the most appropriate bioanalytical strategy, multiple technologies and tools, and various approaches including qualitative and quantitative methods may be needed to understand the impact of biotransformation of biotherapeutics on function.
- Adequate quality of critical reagents should include an early assessment of binding properties (e.g., cross-reactivity to matrix components), binding kinetics and any altered binding issues due to, for example, labeling or storage. Biophysical properties of critical reagents are required by regulators and include identity, concentration, storage conditions and stability information. Purity information is also important.

\section{Biomarkers}

- The best-practices for confirming the specificity of commercially available immunoassays used for clinical biomarker analyses include characterization of the commercial kit. Presence of a signal and sensitivity specifications as determined by the vendor are not enough to confirm that the kit is detecting the biomarker of interest without additional testing; assessing assay specificity is key. Reference standards from different sources should be tested. Parallelism testing should be performed to assess whether recombinant material is comparable to endogenous analyte. If only one kit lot will be used for the entire study, inter-lot variability testing may not be required. However, if multiple lots are needed, it is recommended to establish a lot bridging strategy and test as many lots as reasonably possible to assess risk. If inter-lot variability is observed during study sample analysis, the vendor(s) should be contacted to replace the critical reagents and if not possible, appropriate statistical assessments on the impact of variability and the use of correction factors can be explored to determine the best path forward.

- Exploratory and confirmatory biomarker categories are not always distinct. The application of an assay may evolve during the drug development and the levels of assay validation required may vary depending on the intended use of the data. Due to a variety of challenges, developing and validating the biomarker assay cannot always be performed to meet the standards of a PK assay as requested by the 2013 FDA draft guidance. It is recommended to have at least one QC in the same matrix as the study samples, included among low, medium and high QCs. Stability testing should be performed in both buffer and sample matrix under actual conditions used during sample analysis. Matrix stability should be tested with matrix QC samples where available. Precision and accuracy of a biomarker assay should be assessed. However, when the biomarker methods are relative quantitative or qualita- 
tive, assay validation efforts should be focused on assay precision or reproducibility.

- As for most biomarker assays, reference standards that properly represent endogenous soluble proteins are difficult to find. A fit-for-purpose approach leverages the use of recombinant proteins as reference material. These recombinant reference materials may not be able to fully represent endogenous analytes, which are often heterogeneous and undergo a variety of post-translational modifications such as glycosylation, phosphorylation and oligomerization. Therefore, biomarker assays using recombinant proteins as reference material are deemed relative quantitative in nature. Certain aspects of the reference standards should be assessed to understand what is being measured, including identity (molecular weights and peptide mapping); purity; potency; carbohydrate content; oligomeric and physico-chemical states.

\section{Immunogenicity}

- The use of floating cut-points by pooling the variability across all the assay runs is generally acceptable for screening cut-point evaluations even when the variances are significantly different between assay plates/runs because the use of dynamic cut-points is often impractical. However, as explained in the USP chapter [31], prior to directly implementing the floating cut-point approach, potential causes for the variance heterogeneity should be investigated and re-optimization of the assay should be considered when feasible. For confirmation assays, the fixed cutpoint approach is still the most viable strategy even when the means and/or variances are significantly different across assay plates/runs. Methods for floating cut-point strategy may be considered by spiking the negative control with excess study drug, but this requires further evaluation and validation. Titration cut-points are necessary when the screening cutpoints are too low and fall on the lower plateau of the positive control dilution curve. The cut-points determined during validation may sometimes not be suitable for testing the clinical study samples. If the false positive rate of the clinical baseline samples based on the prestudy validation screening cut-point is under $2 \%$ or over $11 \%$, the use of a study-specific cut-point should be considered.

- Based on current industry and regulatory experience, both one assay and two assay approaches for the demonstration of clinical immunogenic comparability for biosimilars are valid approaches and accepted by regulatory agencies.
- When ADA or target interferes with the PK assays, it is important to understand free drug levels (active drug) to be able to correlate exposure/response. In the ADA assays, false positives and negatives due to target or drug interference are possible; applicable technical modifications should be employed to eliminate this interference and assay caveats should be included in the immunogenicity risk assessment and mitigation plan for appropriate data reporting and interpretation. In the NAb assays, both false positives and false negatives are possible. In these cases, the drug present in the samples should be removed if possible. Interference in and due to drug target (biomarker) should also be considered in the context of these assays.

- Bispecific therapeutic proteins may require additional mechanistic characterizations associated with immune responses in addition to ADA testing such as monitoring cytokine release and other immune system modulation events. Screening and confirmatory assays should minimally be performed against the intact bispecific antibody per conventional testing strategies. Testing for specificity against the functional domain may be justified as the incidence and clinical impact merits. Specificity testing can be done through competitive binding experiments in the confirmatory tier of the testing strategy; alternatively, it can also be achieved by implementing fully independent, domain-based screening assays. The choice of these approaches should be case dependent.

- Cell based and non-cell based assays can be used for the detection of NAbs. Close discussion and interactions with regulators are needed. Kloks's proposal was not fully accepted at this stage [41].

- Pre-existing ADAs are not always due to previous administration of biotherapeutics. In some cases, there is no previous exposure but there is crossreactivity whose nature is not always clear. Hence, observations on the impact of pre-existing antibodies on PK, safety and efficacy are important to know and they can be variable. Patient exclusion based upon pre-existing antibody presence is not recommended. Direct characterization of ADA during the course of therapy should be conducted and in relevant cases, incorporated into the immunogenicity risk assessment approach.

- Monitoring of PK, PD and ADA (and its impact) during the clinical development program, may provide a more comprehensive and personalized approach for disease management post-approval. 
Robust PD biomarker related to efficacy/MOA may be appropriate to correlate available data to clinical impact. An immune response indicator such as antidrug IgG4 antibody, if there is demonstrated impact on safety or efficacy during clinical development and postapproval, may be considered. A conversation with the regulatory agency is recommended in this case.

\section{Acknowledgements}

The authors would like to acknowledge the US FDA, Europe EMA, France ANSM, The Netherlands MEB, UK MHRA, Germany BfArM, Brazil ANVISA, Health Canada, and Japan MHWL for supporting this workshop. E Fluhler (Pfizer), J Welink (EMA/Dutch MEB), B Ackermann (Eli Lilly), N Hughes (Life Labs), F Garofolo (Angelini Pharma), A Song (Genentech), T Thway (Amgen), L Amaravadi (Biogen Idec) and H Myler (Bristol-Myers Squibb) for chairing the workshop and/or the white

\section{Author affiliations}

'Biogenldec, Cambridge, MA, USA

${ }^{2}$ Genentech, South San Francisco, CA, USA

${ }^{3}$ Bristol-Myers Squibb, Princeton, NJ, USA

${ }^{4}$ Amgen, Thousand Oaks, CA, USA

${ }^{5} U S$ FDA, Silver Spring, MD, USA

${ }^{6}$ Abbvie, Souderton, PA, USA

${ }^{7}$ Angelini Pharma, Pomezia, RM, Italy

${ }^{8}$ Roche Pharma Research and Early Development, Roche Innovation

Center, Basel, Switzerland

${ }^{9}$ Sanofi, Framingham, MA, USA

${ }^{10} \mathrm{LGC}$, Cambridge, UK

"Pfizer, Groton, CT, USA

12Pfizer, Andover, MA, USA

${ }^{13}$ Bristol-Myers Squibb, Philadelphia,PA, USA

${ }^{14}$ Novo Nordisk A/S, Maaloev, Denmark

\section{References}

1 Savoie N, Booth BP, Bradley T et al. 2008 White Paper: the 2 nd calibration and validation group workshop on recent issues in good laboratory practice bioanalysis. Bioanalysis 1(1), 19-30 (2009).

2 Savoie N, Garofolo F, van Amsterdam P et al. 2009 White paper on recent issues in regulated bioanalysis from the $3 \mathrm{rd}$ calibration and validation group workshop. Bioanalysis 2(1), 53-68 (2010).

3 Savoie N, Garofolo F, van Amsterdam P et al. 2010 White paper on recent issues in regulated bioanalysis and global harmonization of bioanalytical guidance. Bioanalysis 2(12), 1945-1960 (2010).

4 Garofolo F, Rocci M, Dumont I et al. 2011 White paper on recent issues in bioanalysis and regulatory findings from audits and inspections. Bioanalysis 3(18), 2081-2096 (2011).

5 DeSilva B, Garofolo F, Rocci M et al. 2012 White paper on recent issues in bioanalysis and alignment of multiple guidelines. Bioanalysis 4(18), 2213-2226 (2012). paper discussions. All the workshop attendees and members of the bioanalytical community who have sent comments and suggestions to complete this White Paper. W Garofolo, L Lu, $X$ Wang, M Losauro, N Savoie, A Hernandezand, S Schonert, D Cohen, K Kalaydjian, P de Souza for the assistance in the organization of the event. Future Science Group as a trusted partner.

\section{Financial \& competing interests disclosure}

The authors have no relevant affiliations or financial involvement with any organization or entity with a financial interest in or financial conflict with the subject matter or materials discussed in the manuscript. This includes employment, consultancies, honoraria, stock ownership or options, expert testimony, grants or patents received or pending, or royalties.

No writing assistance was utilized in the production of this manuscript.

\footnotetext{
${ }^{15}$ Celerion, Lincoln, NE, USA
}

${ }^{16}$ Novartis Pharma, Basel, Switzerland

${ }^{17}$ Eurofins Bioanalytical Services, St Charles, MO, USA

${ }^{18}$ Japan MHLW-NIHS, Tokyo, Japan

${ }^{19}$ Eurofins Bioanalytical Services, Abingdon, Oxfordshire, UK

${ }^{20}$ UCB Biopharma, Braine L'Alleud, Belgium

${ }^{21}$ Roche Pharma Research and Early Development, Roche Innovation Center, Penzberg, Germany

${ }^{22}$ Bioagilytix Labs, Durham, NC, USA

${ }^{23}$ CFABS, Laval, QC, Canada

${ }^{24}$ Merck, Rahway, NJ, USA

${ }^{25}$ Regeneron Pharmaceuticals, Tarrytown, NY, USA

${ }^{26}$ Dutch MEB, Utrecht, The Netherlands

${ }^{27}$ Janssen R\&D, Spring House, PA, USA

6 Stevenson L, Rocci M, Garofolo F et al. 2013 White Paper on recent issues in bioanalysis: "hybrid" - the best of LBA \& LC/MS. Bioanalysis 5(23), 2903-2918 (2013).

7 Fluhler E, Hayes R, Garofolo F et al. 2014 White Paper on recent issues in bioanalysis: a full immersion in bioanalysis (Part 1 - small molecules by LCMS). Bioanalysis 6(22), 3039-3049 (2014)

8 Dufield D, Neubert H, Garofolo F et al. 2014 White Paper on recent issues in bioanalysis: a full immersion in bioanalysis (Part 2 - hybrid LBA/LCMS, ELN \& regulatory agencies' input). Bioanalysis 6(23), 3237-3249 (2014).

9 Stevenson L, Amaravadi L, Myler H et al. 2014 White Paper on recent issues in bioanalysis: a full immersion in bioanalysis (Part 3 - LBA and immunogenicity). Bioanalysis 6(24), 3355-3368 (2014)

10 US Department of Health and Human Services, US FDA, center for drug evaluation and research, center for veterinary medicine. Draft Guidance for Industry, Bioanalytical Method Validation, Rockville, MD, USA (2013). www.fda.gov/downloads/drugs/ 
guidancecomplianceregulatoryinformation/guidances/ ucm368107.pdf

11 US Department of Health and Human Services, US FDA, center for drug evaluation and research, center for veterinary medicine. Guidance for Industry, Bioanalytical Method Validation, Rockville, MD, USA (2001). www.fda.gov/downloads/Drugs/.../Guidances/ucm070107. pdf

12 US Department of Health and Human Services, US FDA, Center for Drug Evaluation and Research, Center for Biologics Evaluation and Research. Draft guidance for industry, clinical pharmacology data to support a demonstration of biosimilarity to a reference product, Rockville, MD, USA (2014).

13 US Department of Health and Human Services, US FDA, Center for Drug Evaluation and Research, Center for Biologics Evaluation and Research. Draft guidance for industry, reference product exclusivity for biological products filed under section 351 (a) of the PHS Act, Rockville, MD, USA (2014).

www.fda.gov/downloads/drugs/ guidancecomplianceregulatoryinformation/guidances/ ucm407844.pdf

14 Bellomo G, Sulas MG, Mairate E et al. Hemolysis is a major cause of variability in insulin measurement during oral glucose tolerance test in children. Clin Lab. 58(1-2), 67-74 (2012).

15 Cook PR, Glenn C, Armston A. Effect of hemolysis on insulin determination by the Beckman Coulter Unicell DXI 800 immunoassay analyzer. Clin. Biochem. 43(6), 621-622 (2010).

16 European Medicines Agency. Committee for Medicinal Products for Human Use (CHMP). Guideline on bioanalytical method validation. London, UK (2011). EMEA/CHMP/EWP/192217/2009. www.ema.europa.eu/docs/en_GB/document_library/ Scientific_guideline/2011/08/WC500109686.pdf

17 Spiess C, Zhai Q, Carter PJ. Alternative molecular formats and therapeutic applications for bispecific antibodies. Mol. Immunol. 67(2 Pt. A), 95-106 (2015).

18 Kontermann RE, Brinkmann U. Bispecific antibodies. Drug Discov. Today 20 (7), 838-847 (2015).

19 Staack RF, Stracke JO, Stubenrauch K et al. Quality requirements for critical assay reagents used in bioanalysis of therapeutic proteins: what bioanalysts should know about their reagents. Bioanalysis 3(5), 523-534 (2011).

20 King LE, Farley E, Imazato $M$ et al. Ligand binding assay critical reagents and their stability: recommendations and best practices from the global bioanalysis consortium harmonization team. AAPS J. 16(3), 504-514 (2014).

21 O'Hara DM, Theobald V, Clements Egan A et al. Ligand binding assays in the 21st century laboratory: recommendations for characterization and supply of critical reagents. AAPS J. 14(2), 316-328 (2012).

22 O'Hara DM, Theobald V. Life cycle management of critical ligand-binding reagents. Bioanalysis 5(21), 2679-2696 (2013).

23 Geist BJ, Clements EA, Yang TY et al. Characterization of critical reagents in ligand-binding assays: enabling robust bioanalytical methods and lifecycle management. Bioanalysis 5(2), 227-244 (2013).

24 Khan M, Bowsher RR, Cameron M et al. Recommendations for adaptation and validation of commercial kits for biomarker quantification in drug development. Bioanalysis 7(2), 229-242 (2015).

25 Hurko O. The uses of biomarkers in drug development. Biomarkers in brain disease.. Ann. N.Y. Acad. Sci. 1180, 1-10 (2009).

26 Nowatzke W, Cole TG, Bowsher RR. Systematic analytical validation of commercial kits for determination of novel biomarkers in clinical drug development. Bioanalysis 2(2), 237-247 (2010).

27 Lee JW, Devanarayan V, Barrett YC et al. Fit-for-purpose method development and validation for successful biomarker measurement. Pharm. Res. 23(2), 312-328 (2006).

28 Mire-Sluis AR, Barrett YC, Devanarayan V et al. Recommendations for the design and optimization of immunoassays used in the detection of host antibodies against biotechnology products. J. Immunol. Meth. 289, 1-16 (2004).

29 Shankar G, Devanarayan V, Amaravadi L et al. Recommendations for the validation of immunoassays used for detection of host antibodies against biotechnology products. J. Pharm. Biomed. Anal. 48, 1267-1281 (2008).

30 General Chapter $<1106>$ Immunogenicity assays- design and validation of immunoassays to detect anti-drug antibodies, US Pharmacopeia and the national formulary (USP-NF) (2014).

31 General Chapter <1106> Immunogenicity Assays- Design and Validation of Assays to Detect Anti-Drug Neutralizing Antibody, U.S. Pharmacopeia and the National Formulary (Pharmacopeial Forum 40(3), online May-June 2014.

32 Smith HW, Moxness M, Marsden R. Summary of confirmation cut point discussions. AAPS J. 13(2), 227-229 (2011).

33 Shankar G, Arkin S, Cocea L et al. Assessment and reporting of the clinical immunogenicity of therapeutic proteins and peptides-harmonized terminology and tactical recommendations. AAPS J. 16(4), 658-673 (2014).

34 U.S. Department of Health and Human Services Food and Drug Administration Center for Drug Evaluation and Research (CDER) Center for Biologics Evaluation and Research (CBER) - US FDA Guidance for Industry: Scientific Considerations in Demonstrating Biosimilarity to a Reference Product (2015).

www.fda.gov/downloads/ DrugsGuidanceComplianceRegulatoryInformation/ Guidances/UCM291128.pdf

35 Neubert H, Grace C, Rumpel K, James I. Assessing immunogenicity in the presence of excess protein therapeutic using immunoprecipitation and quantitative mass spectrometry Anal. Chem. 80, 6907-6914 (2008).

36 Mikulskis A, Yeung D, Subramanyam M, Amaravadi L. Solution ELISA as a platform of choice for development of robust, drug tolerant immunogenicity assays in support of drug development. J. Immunol. Methods 365(1-2), 38-49 (2011). 
37 Lee JW, Kelley M, King LE et al. Bioanalytical approaches to quantify "total" and "free" therapeutic antibodies and their targets: technical challenges and PK/PD applications over the course of drug development. AAPS J. 13(1), 99-110 (2011).

38 Sailstad JM, Amaravadi L, Clements-Egan A et al. A white paper-consensus and recommendations of a global harmonization team on assessing the impact of immunogenicity on pharmacokinetic measurements. AAPS J. 16(3), 488-498 (2014).

39 US Department of Health and Human Services, US FDA, Center for Drug Evaluation and Research, Center for Biologics Evaluation and Research. Guidance for industry, Immunogenicity assessment for therapeutic protein products, Rockville, MD, USA (2014).

www.fda.gov/downloads/drugs/ guidancecomplianceregulatoryinformation/guidances/ ucm338856.pdf

40 Gorovits B, Wakshull E, Pillutla R et al. Recommendations for the characterization of immunogenicity response to multiple domain biotherapeutics. J. Immunol. Methods 408, 1-12 (2014).

41 Kloks C, Berger C, Cortez Pet al. A fit-for-purpose strategy for the risk-based immunogenicity testing of biotherapeutics: a European industry perspective. J. Immunol. Meth. 417, 1-9 (2015).

42 US Department of Health and Human Services, US FDA, Center for Drug Evaluation and Research, Center for Biologics Evaluation and Research. Draft guidance for industry. Assay development for immunogenicity testing of therapeutic proteins, Rockville, MD, USA (2009). www.fda.gov/downloads/drugs/ guidancecomplianceregulatoryinformation/guidances/ ucm338856.pdf

43 Bjerner J, Olsen KH, Bormer OP et al. Human heterophilic antibodies display specificity for murine IgG subclasses. Clin. Biochem. 38(5), 465-472 (2005).

44 Watanabe M, Uchida K, Nakagaki K et al. High avidity cytokine autoantibodies in health and disease: pathogenesis and mechanisms. Cytokine Growth Factor Rev. 21, 263-273 (2010).

45 Myler H. "Unresolved issues on pre-existing antibodies and immunogenicity risk." Presented at: 9th Workshop on Recent Issues in Bioanalysis. Miami, FL, USA, 14-16 April 2015.

46 Zhou L, Hoofrig S, Wu Y et al. Stratification of antibodypositive subjects by antibody levels reveals impact of immunogenicity on PK. AAPS J. 15, 30-40 (2012).

47 Banugaria SG, Prater SN, Ng YK et al. The impact of antibodies on clinical outcomes in diseases treated with therapeutic protein: lessons learned from infantile Pompe disease. Genet. Med. 13, 729-736 (2011).

48 Bartelds GM, Krieckaert CL, Nurmohamed MT et al. Development of antidrug antibodies against adalimumab and association with disease activity and treatment failure during long-term follow-up. JAMA 305(14), 1460-1468 (2011).

49 Weeraratne D, Kuck A, Chirmule N, Mytych DT. The measurement of anti-ESA IgG4 antibody as an indicator of antibody-mediated PRCA. Clin. Vaccine Immunol. 20(1), 46-51 (2013). 